\title{
Words in the brain's language: An experimental investigation
}

\author{
Patrizia Setola *, Ronan G. Reilly \\ Department of Computer Science, NUI Maynooth, Maynooth, Co. Kildare, Ireland
}

Accepted 14 December 2004

Available online 10 May 2005

\begin{abstract}
According to Pulvermüller (1999), words are represented in the brain by cell assemblies (Hebb, 1949) distributed over different areas, depending on semantic properties of the word. For example, a word with strong visual associations will be represented by a cell assembly involving neurons in the visual cortex, while a word suggesting action will selectively activate neurons in the motor areas. The present work aims to test the latter hypothesis by means of behavioural measures. Specifically it tests the prediction that there should be a selective influence (in terms either of interference or priming) of performed/observed movements on the performance (reaction times and accuracy) of lexical decision involving words with a strong action association. Similarly, a selective influence of visual images on lexical decision involving words with strong visual associations should be observed. Two experiments were carried out. Results provided partial support for the hypothesis.
\end{abstract}

(c) 2005 Elsevier Inc. All rights reserved.

Keywords: Language; Cell assemblies; Priming

\section{Introduction}

According to a widely established view, language constitutes a mental module (Chomsky, 1988), largely independent of general cognition, where the lexicon acts as a mental dictionary, with each word corresponding to a specific entry. Each entry contains information about the word's meaning, its part of speech, and its relationship with the other words. This view of language has produced several models within the classical symbolic approach, as it readily lends itself to the proposal of theories involving the rule-governed manipulation of tokens. The rules invoked are primarily syntactical. The influence of this traditional view, however, extends beyond classical models, involving also some connectionist approaches. Even if neural networks are proposed as a more neurologically plausible means of representation, overcoming the problematic assumption of complex built-in algo-

\footnotetext{
* Corresponding author. Fax: +3531 7083848.

E-mail address: psetola@cs.may.ie (P. Setola).
}

rithms, words are still conceived as disembodied symbols, connected to each other through associative links of variable strength (e.g., LSA, Landauer \& Dumais, 1997). The idea of semantic memory being represented by nodes, where activation spreads automatically from one node to the nearby ones along associative pathways (Collins \& Loftus, 1975), constitutes an accepted theoretical account of semantic priming (for other accounts, such as backward priming or expectancy generation, see Balota, 1994). Priming is a phenomenon whereby previous exposure to information (the prime), which can be orthographically, phonologically, or semantically related to the target, influences the speed and/or accuracy of word recognition processes in various tasks, such as naming or lexical decision. Within this theoretical framework, facilitation of related words and inhibition of unrelated words are explained in terms of the relative spatial proximity of the nodes (words) in the semantic network. However, the model operates at a descriptive, cognitive level, with little or no connection to the underlying neurological processes. Cognitive neuroscience can help shed some light 
on the way words are actually represented in the brain, beyond the mental dictionary metaphor, providing a more biologically motivated foundation for priming and other related issues.

The main inspiration for the present research arose from two proposals regarding the neurobiological bases of language, supported by an increasing amount of empirical findings. First is the idea that language areas seem to have sensorimotor roots. For example, activation in the Broca's area is observed either when subjects perform complex sequences with the hand and fingers, or when they observe such movements by another person (Rizzolatti \& Arbib, 1998), suggesting that the language faculty may have arisen as an adaptation of the sensorimotor system. Second is the concept that cell assemblies are "possible building blocks of cognition" (Pulvermüller, Preissl, \& Carsten, 1994; Reilly, 2001), and that distributed functional networks underlie all higher cognitive processes, including language.

According to Hebb (1949), cell assemblies are functional units, comprising neurons that are strongly interconnected by excitatory synapses, such that activation of a subset of the population leads to activation of the whole assembly in a self-sustaining process. The cell assembly hypothesis, as proposed by Hebb (1949), is based on several fundamental postulates. (1) The strengths of the connections (the synapses) between two neurons can alter with time: if the pre-synaptic neuron and the post-synaptic neuron are both active at the same time, then the strength of the synapse will increase. (2) The connections between groups of neurons that tend to fire together will strengthen (through a process now known as Hebbian learning), leading to the formation of strongly associated circuits referred to as cell assemblies.

Applying Hebb's model to language, (Pulvermüller \& Preissl, 1991; Pulvermüller et al., 1994; Pulvermüller, 1999, 2001) hypothesised that words are represented by distributed functional neural networks. His crucial contribution is the idea that distributed cell assemblies representing words contain, in addition to neurons in the recognised language areas, also neurons in areas related to aspects of the words' meaning (Pulvermüller, 1999). In fact, during language acquisition 'neurons related to a word form become active together with neurons related to perceptions and actions reflecting aspects of its meaning' (p. 12). Repeated co-activation of neurons in different cortical (or even subcortical) areas leads eventually to the formation of a higher-order assembly, which can be entirely ignited by the activation of any sufficient part.

Over the years Pulvermüller has successfully testedwith behavioural, electrophysiological, and metabolic studies - a number of predictions based on his hypothesis. In particular, in agreement with the idea that assemblies constitute the basic units of word representation, neuronal populations become active when a word is being processed. Cell assembly ignition will take place after presentation of a word but not of a pseudoword, which will induce only partial - if any - activation. (Pulvermüller et al., 1994). Critically, different word categories are represented by cell assemblies with a different topography. On the one hand function words, whose meanings reflect their linguistic use rather than objects or actions, should be represented exclusively by strongly left-lateralised assemblies limited to the perisylvian cortices; on the other hand, content words should be represented by less lateralised assemblies, including neurons both within and outside the perisylvian regions. Within content words a further distinction is expected between 'perception' and 'action' words: vision words, referring to visual stimuli, should induce additional activation in the visual areas (occipital and infero-temporal lobes); action words, referring to movements performable by one's own body, should evoke additional activation in the motor areas (Pulvermüller, 1999).

Developmental (Bates, Bretherton, \& Snyder, 1988) and neuropsychological studies have suggested dissociation between objects and actions, grounded in different underlying brain organisations. While models influenced by traditional theories of grammar (e.g., generative grammar) ascribe the observed differences to their respective syntactic roles (nouns and verbs), Pulvermüller's work offers an alternative explanation using neurally based semantic distinctions. Indeed, the distinction extends beyond lexical boundaries, with nouns associated with actions behaving like verbs (Pulvermüller, 1999), and verbs with no action association behaving like nouns ( $\mathrm{Lu}$ et al., 2002).

Further fine-grained distinctions can be observed within each category, for example, with different topographies for cell assemblies corresponding to leg-, arm-, and face-related words correlated with the somatotopic organisation of the motor cortex (Pulvermüller, 2001).

The crucial idea is that, rather than being nodes within an associative network, words are represented by cell assemblies, which can overlap to various extents, depending on their shared meaning. This hypothesis can provide a more neurobiologically plausible framework for the explanation of priming-like effects. If semantically related words are represented by overlapping assemblies, then activation of the neural population representing a specific word equates to the activation of a subset of the cell assemblies representing semantically related words. In this way, ignition of the cell assembly for the target word is facilitated by exposure to a related prime.

Furthermore, if content words at least involve activation of the sensorimotor areas representing aspects of their meaning, then it is worth investigating whether behaviours other than words (e.g., actions, perceptions, etc.) have an influence on word recognition processes. Again, if the respective cell assemblies overlap, can a performed/observed action facilitate the recognition of 
action words? Alternatively, will interference occur, as different tasks compete for the same neural resources?

In terms of the cell assembly hypothesis, the influence,-if any - of action (either perceived or performed) on action words, of images on vision words, etc., does not need to be mediated by the verbal label, that is, actions/images do not require to be named in order for the influence to occur. Rather, the influence should be direct, and depend on recruited overlapping cell assemblies. In contrast with the traditional view of priming, therefore, not only nameable, but also non-nameable actions should have an influence on speed and/or accuracy of word recognition.

\section{Experiment 1}

The following experiment was carried out to investigate the hypothesis that the functional unit of the brain representing action words is a cell assembly involving additional neurons in the motor areas (Pulvermüller, 1999).

Specifically, it was aimed at testing the prediction that there should be a selective influence, either in terms of interference or priming, of performed movements on the recognition of action words describing the movements, in contrast to both general action words and pseudowords, due to the simultaneous activation of overlapping cell assemblies. Moreover, the expected effect should be detectable with behavioural measures, such as speed and accuracy in performing a lexical decision task.

Two hypotheses were tested. First that movements performed by hand would have a selective influence on reaction times and errors for lexical decision on words describing actions performed by hand, as opposed to general action words or pseudowords. Second, the "nameability" of actions would also influence performance, enhancing RTs and/or accuracy as a function of semantic priming. It was predicted that nameable actions would speed reactions and reduce errors for actual action words compared to other word categories.

\section{Procedure}

Eighteen native English speakers performed lexical decisions on visually presented words and matched pseudowords (a global set of 48 stimuli, reported in Appendix A), of which: 32 English words matched for length (four letters) and frequency (the words chosen had a frequency range of 10-191 per million, from Leech \& Rayson, 2001); and 16 pseudowords (PW) matched for length (four letters), phonologically legal, and derived from 16 of the actual words by changing only one character, (e.g., pull $\rightarrow$ 'pule'). The 32 actual words were verbs strongly associated with action, half of them

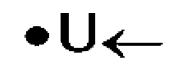

Fig. 1. One of the 16 symbols used as cues in Experiment 1. This one represents the action "pull."

describing actions performable with one's hand (or hand action words, HAw; e.g., pull, push, etc.); and half describing general actions (or general action words, GAw: e.g., view, wake, etc).

Following a training session, one of 16 iconic visual cues (see Fig. 1) appeared in black at the centre of a white computer screen, indicating that the associated action was to be performed. Each visual cue was consistently associated with one of 16 simple actions, which were of two different types: eight actions describable with a single word (nameable), acting as appropriate cues for the linked hand action words; eight not describable with a single word (un-nameable), and acting as inappropriate cues for the linked hand action words.

The cue lasted on the screen for $1000 \mathrm{~ms}$, and was immediately followed by the appearance of one of the three linked words (a HAW, a GAW, or a PW; again black letters on a white background) in place of the symbol. While still performing the cued action with the left hand, subjects had to decide whether the stimulus was a real word or not, clicking with their right hand, respectively, on the left or the right mouse button. An intervening stimulus, in the form of a black rectangle at the centre of the screen, was presented after each lexical decision. To proceed, the middle mouse button had to be clicked. This way, subjects could adapt the task completion at their own pace. Each subject was presented once with the whole block of 48 words, one at a time, and in random order.

Reaction times were recorded from the appearance of the word on the screen to the response. A record was also kept of subject responses.

The two independent variables, type of word, with three levels (HAW, GAW, and PW) and nameability of the action, with two levels (nameable, un-nameable) were both within subjects. This gave a balanced $3 \times 2$ design, with a total of six combined conditions. Reaction times and errors for the different levels of the two independent variables were analysed using a 3 (word type) $\times 2$ (action type) ANOVA for repeated measures.

\section{Results}

\subsection{Reaction times}

Reaction times for the lexical decision task for each participant on each trial were recorded. The mean RTs for the three types of word and the two kinds of action are displayed in Fig. 2. 


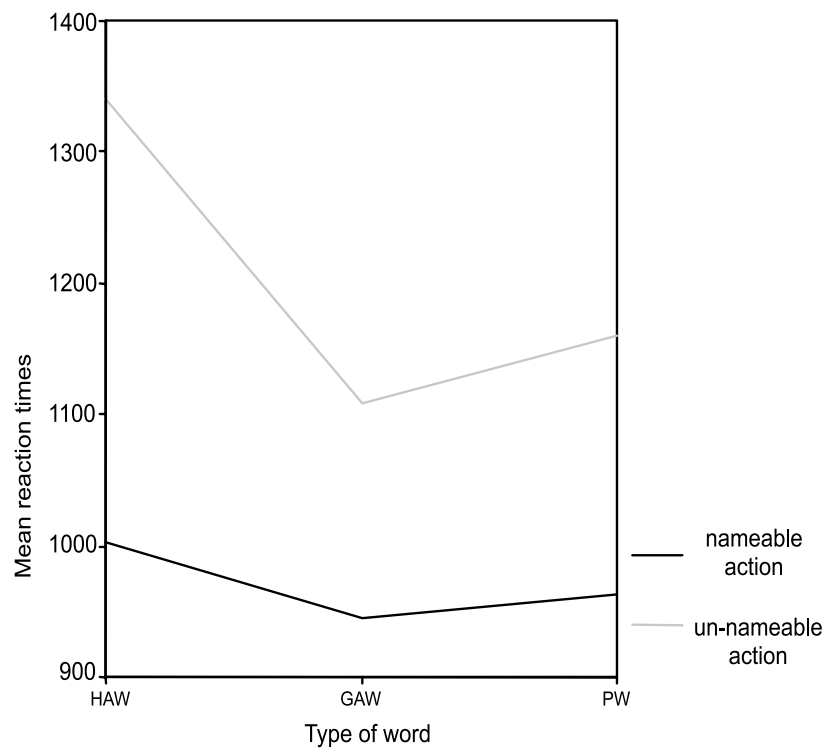

Fig. 2. Reaction times by action type and word type from Experiment 1.

In a repeated measures ANOVA, the main effect for type of word $(F(2,34)=1.946, p>.05)$, and the interaction between type of word and type of action $(F(2,34)=0.724, p>.05)$ were not significant. However, the main effect of type of action $(F(1,17)=9.857, p<.01)$ was significant; slower reaction times resulted when any kind of word was preceded by an un-nameable action, rather than a nameable one.

\subsection{Errors}

The percentage of errors for the lexical decision task for each participant on each trial was recorded. The error percentages for the three types of word and the two kinds of action are shown in Fig. 3.

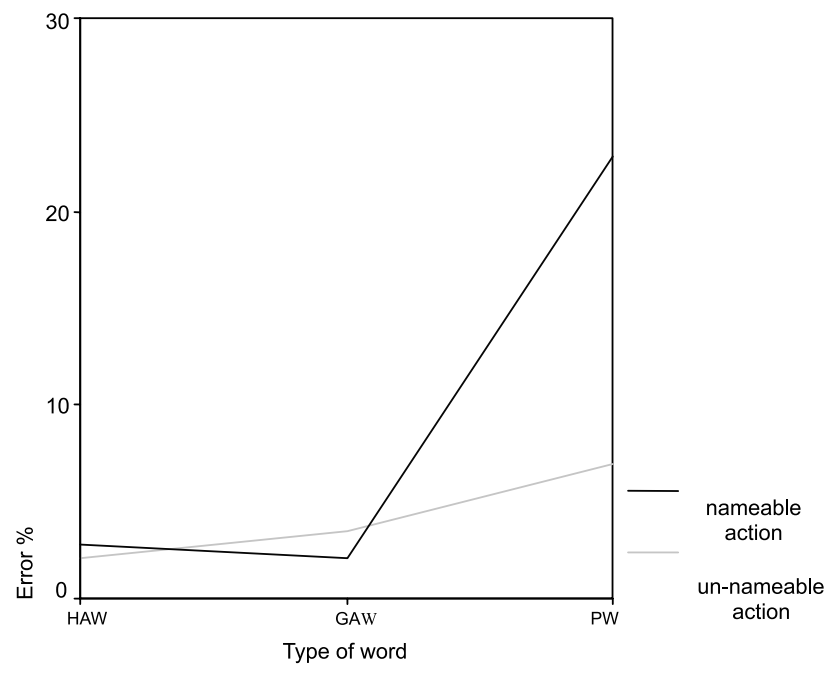

Fig. 3. Percentage of errors in Experiment 1 for HAw, GAw, and PW presented, respectively, after a nameable and an un-nameable action.
A repeated measures ANOVA on the percentage of errors produced a significant main effect for type of word $(F(2,34)=20.118, p<.01)$; many more errors were made for pseudowords than actual words. The main effect of nameability of action was significant $(F(1,17)=13.714, p<.01)$; nameable actions produced more errors. The interaction between type of word and type of action was also significant $(F(2,34)=, p<.01)$, with the highest percentage of errors for pseudowords preceded by nameable actions.

\section{Discussion}

\subsection{Reaction times}

According to the first hypothesis, there should be a differential effect of performed movements on lexical decision times for different types of words. Although without reaching statistical significance, results display a meaningful pattern with the fastest RTs for general action words, slightly slower for pseudowords and even slower for hand action words.

The second hypothesis that nameable and un-nameable actions should have a different influence on the reaction times in the lexical decision task was supported by the statistical analysis, and the difference was highly significant. This result is open to several interpretations. The classic explanation would be in terms of semantic priming: the name given by the subject to the movement performed acted as a prime for the subsequent word. However, nameability was found to affect significantly reaction times for pseudowords as well. While words were controlled for frequency, the actions performed weren't. It might be the case that nameable actions are more frequent than un-nameable ones, therefore this result could be reducible to an action frequency effect.

The interaction between nameability and type of action was not statistically significant, suggesting that nameability influenced all the three-presented types of stimulus in the same way. In fact, there was an increase in the subjects' reaction times for both action words and pseudowords when they were preceded by un-nameable actions.

One possible explanation is that the process of identifying un-nameable actions from the icons presented on the screen and then executing the actions might have proved cognitively more demanding than their nameable counterparts. So in Fig. 1, we are seeing two effects, an overall cognitive load effect across all action types and an interference effect specifically affecting hand actions.

\subsection{Errors}

In agreement with the first hypothesis that there should be a differential effect of performed movements 
on the percentage of lexical decision errors for different types of words, many more errors were made for pseudowords than for actual words (both HAw and GAw).

The second hypothesis that nameable and un-nameable actions should have a differential influence on accuracy was also supported: fewer mistakes were made for un-nameable actions compared to nameable actions.

The interaction between the two variables was also significant with an increase in the percentage of errors for pseudowords than for words in the nameable condition.

The result appears consistent with the assumption that the movement performed (e.g., the action of pulling) will activate part of the cell assembly (the prefrontal subset) representing the word that describes the action (e.g., 'pull') and the semantically related words. The associated pseudoword (e.g., 'pule') may partially activate the cell assemblies representing orthographically related words (Pulvermüller et al., 1994). Among the partially activated words, it will be likely to find the name given to the action performed (pull). Simultaneous activation of subsets of the same cell assembly (representing the word 'pull'), could cross the ignition threshold and lead to activation of the entire assembly. The expected result being either a slower decision, to inhibit the wrongly activated cell assembly or, alternatively, more errors: pseudowords will be more easily judged as real words when accompanied by nameable movements (see Fig. 4).

The latter is exactly the result observed: the performance of subject for pseudowords accompanied by nameable movements was less accurate. The string 'snir,' in particular, was systematically misjudged as a word by all the 18 subjects tested when accompanied by the action of stirring.

In summary, the results of Experiment 1 are equivocal with respect to the possibility of direct priming of words
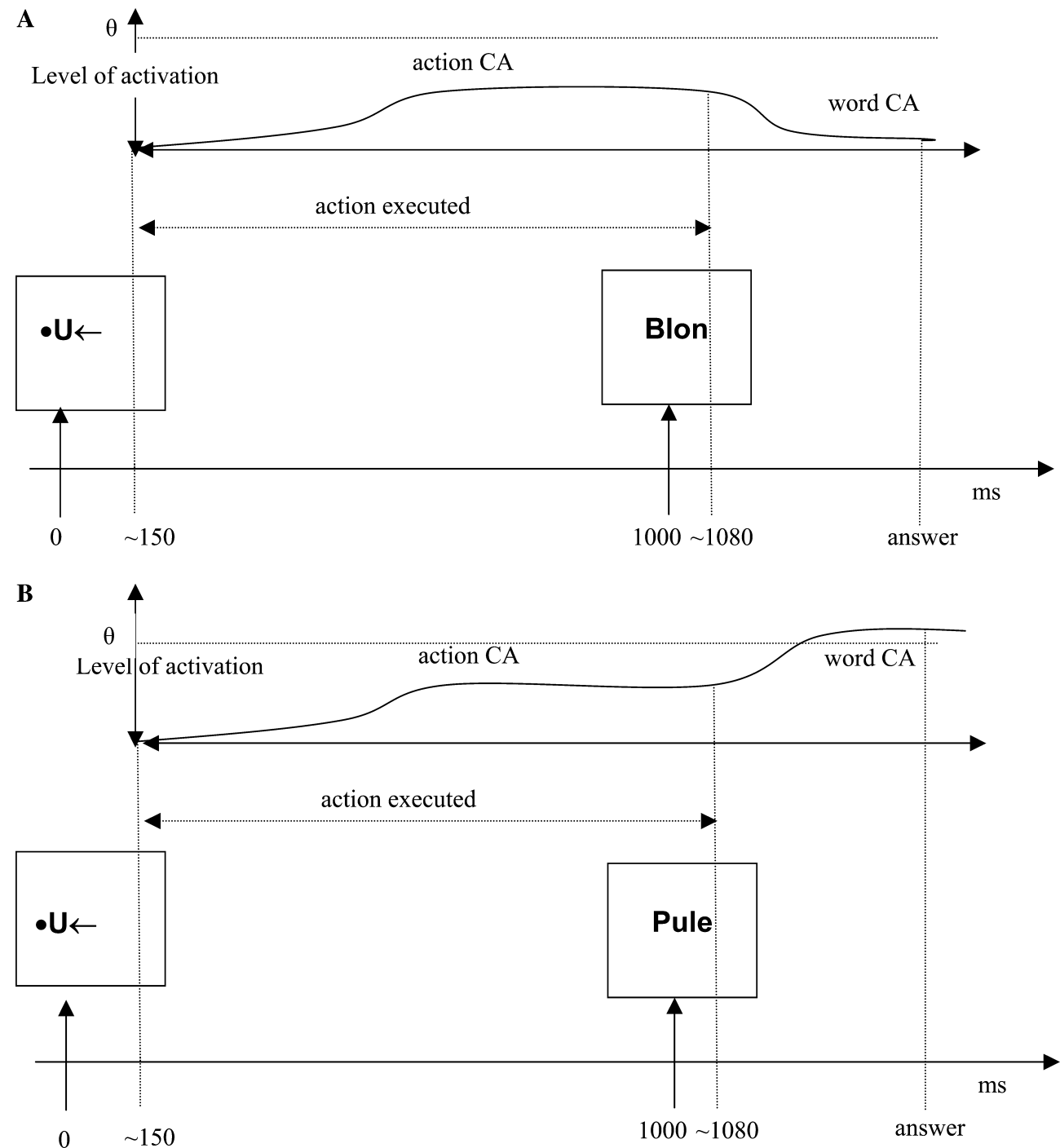

Fig. 4. An 'inappropriate' pseudoword following a performed action is correctly judged as a non-word, as it fails to activate any word cell assembly (A); an 'appropriate' pseudoword (e.g., pule), however, can lead to ignition of the cell assembly corresponding to the related word (pull), and therefore be misjudged as a real word (B). 
by related actions. We have demonstrated, however, that actions significantly influence lexical decisions on semantically related words, but the effect is primarily one of interference, with un-nameable non-cueing actions increasing overall reaction times, and also having an especially large effect on hand action words. The interpretation of these data is also complicated by the fact that the task of icon interpretation may have placed an additional cognitive burden on the subjects and obscured some early onset cell assembly activation effects.

\section{Experiment 2}

Experiment 2 was designed to address a number of shortcomings in the preceding experiment. The most important one being the confound between appropriateness and nameability; in Experiment 1, nameable actions always acted on hand action words and the matching pseudowords as appropriate cues, while un-nameable actions always acted on hand action words and the matching pseudowords as inappropriate cues. This is for the obvious reason that there could never be, by definition, a word for which an un-nameable action was a cue. Therefore, two 'appropriateness' conditions were created: (1) words and matching pseudowords were preceded by an appropriate cue; (2) words and matching pseudowords were preceded by an inappropriate cue. In fact, a testable prediction is that pseudowords should be more likely to be judged as real words in Condition 1 than in Condition 2, where no direct priming could occur.

In this way, the two variables nameability and appropriateness are orthogonal: all the cues considered are nameable, and used in the two conditions once as appropriate and the other as inappropriate cues.

Second, hand actions and corresponding hand action words were tested against images and corresponding vision words, to address also the perceptual half of the sensorimotor domain. An additional reason for the introduction of vision words was the observation that in Experiment 1 reaction times didn't seem to capture the more subtle distinction between hand actions and general actions.

Finally, the symbols used in Experiment 1 to prompt action were substituted by video-clips of performed actions, to make them comparable with the images used as prompts for vision words. Also, in this way, the unnecessary layer of symbol interpretation was eliminated, rendering the experiment more direct and easier for the subjects.

On the basis of Pulvermüller's hypothesis, there should be a selective influence of observed movements on the recognition of action words describing the same movements, in contrast to both vision words and pseudowords, due to the simultaneous activation of overlapping cell assemblies. Similarly, there should be a selective influence of visual images on vision words, compared to action words and pseudowords. Again, the expected effect should be detectable with behavioural measures, such as recorded reaction times (RTs) and response accuracy during a lexical decision task. Implicit in the design of the experiment is the assumption that viewing actions will activate motor neurons involved in carrying out the actions (Rizzolatti \& Arbib, 1998).

Two hypotheses were tested. First that observed hand movements (in the form of brief video-clips) would have a selective influence on reaction times/ errors at the lexical decision for words describing actions performed by hand, as opposed to vision words and pseudowords. This prediction rests on the idea that an action would activate the same, 'mirror' neurons, regardless of the fact that it is performed or observed (Rizzolatti \& Arbib, 1998). Second, the 'appropriateness' of the cue would also influence the performance, enhancing RTs, and/or accuracy as a function of semantic priming. Specifically, more positive errors should emerge from the subjects' performance when pseudowords are preceded by the appropriate cue, compared to inappropriate cues.

\section{Procedure}

Seventeen native English speakers performed lexical decisions on visually presented words and matched pseudowords (a global set of 48 stimuli, reported in Appendix B), of which: 32 English words matching for length (four and five letters evenly distributed among conditions) and frequency (from Leech \& Rayson, 2001), and 16 pseudowords (Pw) matching for length, phonologically legal, and derived from 16 of the actual words changing only one character (e.g., blue $\rightarrow$ 'blee'). Of The 32 actual words, 16 were verbs strongly associated with action performable with one's hand (or hand action words, HAw; e.g., pull, push, etc.), and 16 were nouns/ adjectives describing visual features (vision words, vw: e.g., blue, dark, etc.).

Preceding each word, one of 16 visual cues appeared inside a rectangle at the centre of a white computer screen. Visual cues were of two different types: eight video clips of recorded hand actions, acting as proper cues for the linked hand action words; eight visual images (of both objects and non-objects), acting as proper cues for the linked hand action words.

Each cue lasted on the screen for $1200 \mathrm{~ms}$, independently from the category to which it belonged, and was immediately followed by the appearance of one of the three linked words (a HAW, a Vw, or a Pw; black letters on a white background) in place of the image. Subjects had to decide as fast and accurately as possible, whether the 
stimulus was a real word or not, clicking with their right hand, respectively, on the left or the right mouse button. An intervening stimulus, in the form of a black rectangle appearing at the centre of the screen, was presented after each lexical decision. To proceed, the space bar had to be pressed. This way, subjects could adapt the task completion at their own pace. Each subject was presented once with the whole block of 48 words, one at a time, and in random order.

Reaction times were recorded from the appearance of the word on the screen to the response. A record was kept also for correct and incorrect answers.

The two independent variables, respectively, type of word, with three levels (HAw, vw, and PW) and type of cue, with two levels (action vs. image and proper vs. improper cue) were both within subjects. A balanced $3 \times 2$ design resulted with a total of six combined conditions. Reaction times and errors for the different levels of the two independent variables were analysed using a 3 (word type) $\times 2$ (cue type) ANOVA for repeated measures.

\section{Results}

\subsection{Reaction Times}

Reaction times for the lexical decision task for each participant on each trial were recorded. The mean RTs for the three types of word and the two kinds of cue (action vs. image) are displayed in Fig. 5.

Carrying out an analysis of variance for repeated measures, the main effect for type of word $(F(2,32)=29.455, p<.01)$ was significant. According to the Bonferroni test, the source of the significant difference was the difference between real words and pseudowords. The main effect for type of cue and the

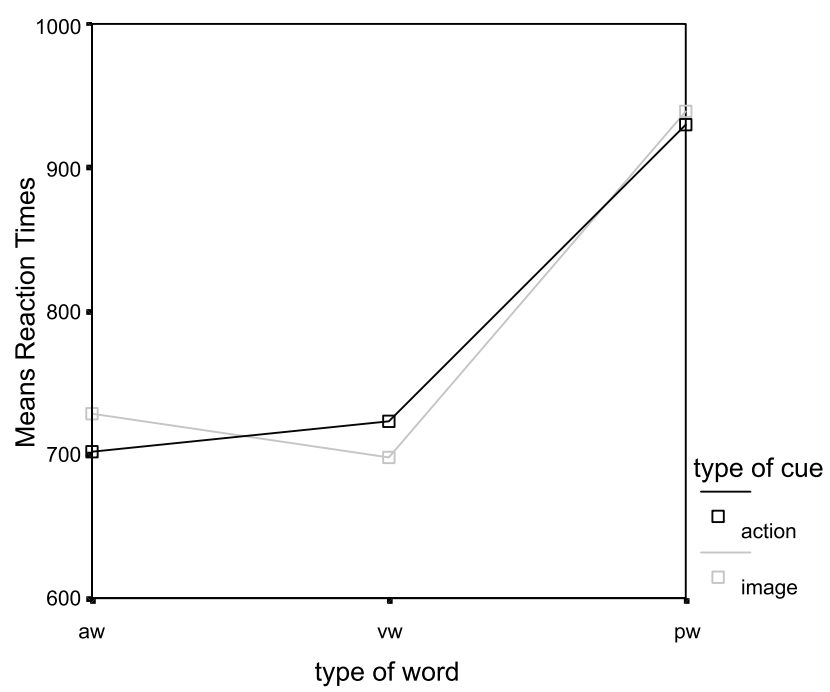

Fig. 5. Reaction times recorded by type of cue (action vs. image) and word type in Experiment 2.

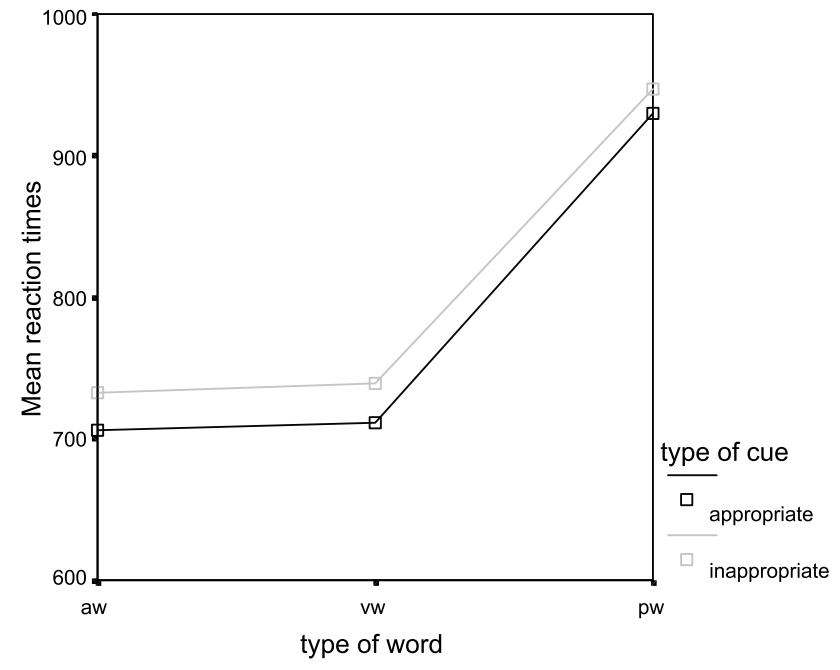

Fig. 6. Reaction times recorded by type of cue (appropriate vs. inappropriate) and word type in Experiment 2.

interaction between type of word and type of cue were not significant. Pseudowords always required the highest RTs, regardless of the kind of cue.

The mean RTs for the three types of word and the two kinds of cue (appropriate vs. inappropriate) are displayed in Fig. 6.

The analysis of variance produced a significant main effect for type of cue $(F(1,16)=7.382, p<.05)$. The inappropriate cue was related to slower reaction times. The main effect for type of word $(F(2,32)=28.701, p<.05)$ was also significant. Again, pseudowords required the slowest reaction times. The interaction between type of word and type of action was not significant.

\subsection{Errors}

The percentage of errors for the lexical decision task for each participant on each trial was recorded. The error percentages for the three types of word and the two kinds of cue (appropriate vs. inappropriate) are shown in Fig. 7.

From the analysis of variance, the main effect for type of word was found to be significant $(F(2,30)=8.730$, $p<.01)$ : many more errors were made for pseudowords than actual words. The quadratic component of the interaction between the kind of word and the kind of cue $(F(2,30)=3.824, p<.05)$ was also significant with the highest percentage of errors for pseudowords in the appropriate condition.

The error percentages for the three types of word and the two kinds of cue (action vs. image) are shown in Fig. 8.

The analysis of variance produced a significant main effect for type of word $(F(2,32)=7.667, p<.01)$ : many more errors were made for pseudowords than actual words. The main effect for type of cue (action vs. image) was not significant $(F(1,16)=0.845, p>.5)$. 


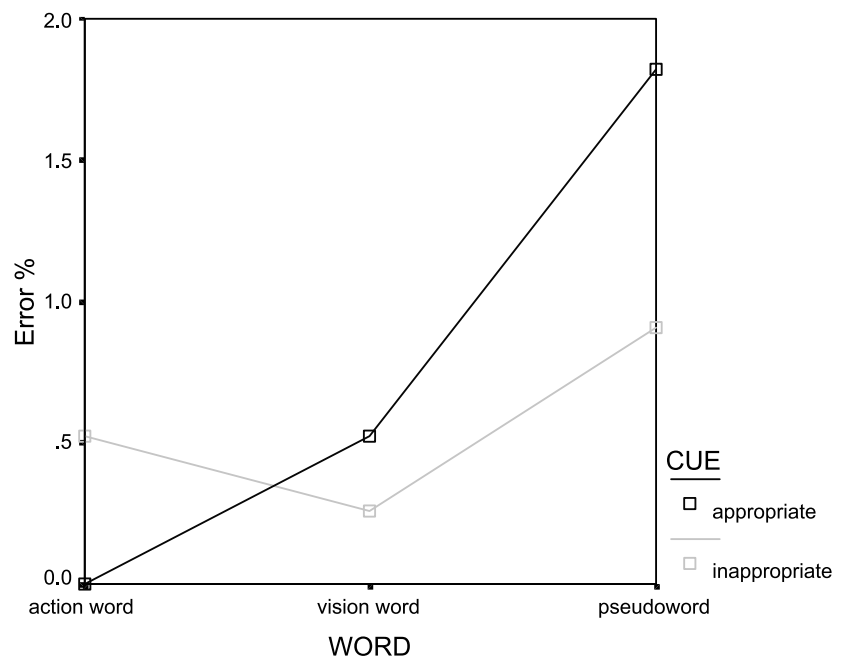

Fig. 7. Percentage of errors in Experiment 2 for HAw, vw, and PW presented, respectively, after an appropriate and an inappropriate cue.

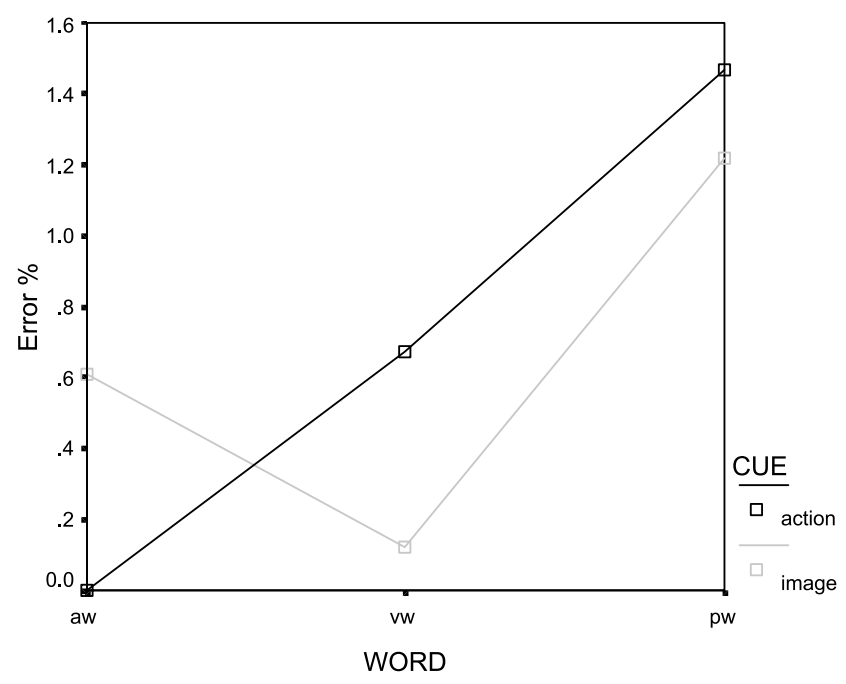

Fig. 8. Percentage of errors in Experiment 2 for HAw, vw, and PW presented, respectively, after an action and an image as cues.

\section{Discussion}

\subsection{Reaction times}

Reaction times, once again, seemed to capture only the well-documented difference between actual words and pseudowords, while contrary to the first hypothesis no significant difference was found between the reaction times for the two different types of actual words (action vs. image). The RTs globally failed to reflect results from functional neuro-imaging studies. However, reaction times were consistently slower for any kind of word when preceded by inappropriate cues as opposed to appropriate cues. Therefore, inappropriate cues seem to impede lexical decision.

\subsection{Errors}

The pattern emerging in subjects' errors is more interesting: the error percentage for pseudowords is much higher in the appropriate condition, compared to the inappropriate condition. Pseudowords seem more likely to be misjudged as real words when 'appropriately' cued by an action/image. Indeed, subjects were more likely to judge the pseudoword as a real word when the pseudoword was preceded by an image/action describable with the word from which it was derived (for example, press $\rightarrow$ 'presh').

This result provides stronger evidence in support of the outcome of Experiment 1, since Experiment 2, clearly distinguished between the variables 'nameability' and 'appropriateness.'

Interestingly, the same pattern of errors emerging for both performed and observed actions lends support to the existence of mirror neurons in humans (Rizzolatti \& Arbib, 1998).

Taken together, the two experiments provide support for the proposal that performed/observed movements, and visual images may be direct partial primes for words with strong action and vision associations, respectively. However, as the actions presented were all nameable it could still be possible to resort to the classical explanation of priming arising from the movements being named, with the name then acting as a prime. This argument is less tenable in the case of the visual images, since the subject could not know in advance which feature of the image would be referred to by the following word. Furthermore, images and actions did not differ significantly in terms of the number of lexical decision errors on the target words/pseudowords.

These results, without entirely ruling out the classical view on priming, suggest the possibility of a broader account of priming effects in terms of cell assemblies. From this perspective, a symbol (e.g., word or pseudoword) is just one of a number of sources of activation that can lead to the ignition of a cell assembly associated with a given concept.

\section{Appendix A}

List of the 48 four-letter strings used in Experiment 1

\begin{tabular}{lll}
\hline Hand action words & General action words & Pseudowords \\
\hline pull & rise & pule \\
push & feed & pesh \\
drop & ride & brop \\
grab & blow & gral \\
shut & race & shub \\
wave & flow & wame \\
stir & leap & snir \\
rest & spin & relt \\
chop & grow & rive \\
snap & fill & ferd \\
toss & grin & nide \\
pick & step & blon \\
fold & lean & ract
\end{tabular}




\section{Appendix A (continued)}

\begin{tabular}{lll}
\hline Hand action words & General action words & Pseudowords \\
\hline lift & wake & flod \\
curl & dive & lerp \\
stay & view & spip \\
\hline
\end{tabular}

\section{Appendix B}

List of the 48 four-letter strings used in Experiment 2

\begin{tabular}{lll}
\hline 16 Vision words & 16 Action words & 16 Pseudowords \\
\hline grey & drop & brop \\
blue & grab & gral \\
pink & push & stap \\
flag & pull & presh \\
dark & shut & swink \\
ring & snap & twilt \\
arch & wave & shub \\
half & bend & frace \\
green & throw & grel \\
brown & shake & blee \\
rough & swing & rint \\
round & twist & jalf \\
shape & press & gruen \\
thick & touch & browl \\
sharp & knock & shafe \\
white & trace & whime \\
\hline
\end{tabular}

\section{References}

Balota, D. (1994). Visual Word Recognition. In Handbook psycholinguistics. MA, Gernsbacher: Academic Press Inch.
Bates, E., Bretherton, I., \& Snyder, L. (1988). Form first words to grammer: Individual differences and dissociable mechanisms (p. 326). New York: Cambridge University Press.

Chomsky, N. (1988). Generative Grammar: Its Basis, Development and Prospects. Studies in English Linguistics and Literature, Special Issue, Kyoto University of Foreign Studies.

Collins, \& Loftus (1975). A spreading activation theory of semantic memory. Psychological Review, 82, 407-428.

Hebb, D. O. (1949). The Organisation of Behaviour. New York: Basic Books.

Landauer, T. K., \& Dumais, S. T. (1997). A solution to Plato's problem: The Latent Semantic Analysis theory of acquisition, induction and representation of knowledge. Psychological Review, 104(2), 211240

Leech, G. N., \& Rayson, P. (2001). Word frequencies in written and spoken language: Based on the British national corpus. London: Longman.

Lu, L. H., Crosson, B., Nadeau, S. E., Heilman, K. M., Gonzalez-Rothi, L. J., Raymer, A., et al. (2002). Category-specific naming deficits for objects and actions: Semantic attribute and grammatical role hypothesis. Neuropsychologia, 1381, 1-14.

Pulvermüller, F., \& Preissl, H. (1991). A cell assembly model of language. Network, 2, 455-468.

Pulvermüller, F., Preissl, H., Carsten, E. (1994). Brain rhytms, cell assemblies and cognition: Evidence from the processing of words and pseudowords. Psycholoquy.

Pulvermüller, F. (1999). Words in the brain's language. Behavioural and Brain Sciences, 22, 253-336.

Pulvermüller, F. (2001). Brain reflections of words and their meaning. Trends in Cognitive Science, 5(12).

Reilly, R. (2001). Collaborative cell assemblies: Building blocks of cortical computation. In S. Wermter, J. Austin, \& D. Willshaw (Eds.), Emergent neural computational architectures based on neuroscience. Heidelberg: Springer-Verlag.

Rizzolatti, G., \& Arbib, M. A. (1998). Language within our grasp. Trends in Neuroscience, 21(5), 188-194. 